\title{
The aftermath of a radiation accident
}

\author{
Kelly Erickson Goldman • Javad Rahimian • Rosalie Kim • \\ Francois Eschwege $\cdot$ A. Robert Kagan
}

Received: 23 May 2014 / Accepted: 13 August 2014 / Published online: 21 August 2014

(C) Springer-Verlag Berlin Heidelberg 2014

As no amount of preparation can render one as fully equipped for an earthquake or a tsunami and so it is that nothing learned can help a radiation oncologist effectively handle the threats of a serious mishap during the treatment of a patient. Despite our comprehensive and exhaustive informed consent process, it serves no weight for the patient and family when the incident occurs. The field of radiation oncology is evolving at a breakneck pace. The most common sources of errors in the past decades have now been addressed with implementation of the transfer of patient data into the record and verify system and the widespread replacement of manual blocking with the multi-leaf collimator. As our techniques have evolved, our quality assurance measures have developed certainly. Our physicists tirelessly perform quality control measures and our radiation therapists' pre-treatment checklists and mandated time-outs grow in length and complexity. All of these have undoubtedly resulted in fewer radiation injuries over the years. Nonetheless, the increasing complexity of our techniques, the rapidity with which we can deliver treatment, and the high monitor units delivered within minutes all compound the probability that a routine error may turn into a disaster.

We have become familiar with few yet devastating errors not through our own literature but primarily through their publication in national newspapers [1-3]. An incident in which an intensity-modulated radiation therapy (IMRT) treatment was delivered with an open field highlighted an

\footnotetext{
K. E. Goldman $(\bowtie) \cdot R$. Kim

Kaiser Permanente Los Angeles Medical Center, Los Angeles, CA, USA

e-mail: kelly.t.erickson@kp.org

J. Rahimian • A. R. Kagan

Department of Radiation Oncology, Southern California Permanente

Medical Group, Los Angeles, CA, USA

F. Eschwege

Gustave-Roussy Institute, Radiation Oncology, Villejuif, France
}

oversight in which computer software was unable to neutralize the issue of human error [1]. Over the years, we, as a field, have seen inaccuracies in calibration, mistakes at commissioning of treatment planning, incorrect site identification and treatment delivery, and incorrect prescription points carried out to treatment on high-dose brachytherapy cases. Even in the absence of a systematic error in the treatment delivery, the concept of an LD 5/5 demands that we recognize and accept, on behalf of our patients, is a complication rate of $5 \%$. Regardless of the cause, a bad outcome for the patient may be perceived similarly and demands attention.

To realize how devastating the result of the radiation injury-whether it be colostomy, permanent incontinence, fistula - can be to a patient's quality of life and independence is only possible if one experiences it. Reading about it is not sufficient. Patients, who previously placed their trust and confidence in their doctor along with the treatment and technology, may now find their lives compromised. Many of them reported organ dysfunction during treatment, which was dismissed as tolerated or expected. They are puzzled at how such a serious mistake could happen when their chart was checked weekly and their images reviewed daily. They feel betrayed by the technology and the team that previously instilled such confidence. No matter what the facts and circumstances, patients see the radiation oncologist as the responsible party.

We, as physicians, are taught to heal and are ill-prepared for the guilt, stress, and discomfort of confronting the error. To avoid causing pain to the patient and ourselves, we may use the justification that "this is the price of treatment." Uttering the words "unexpected" or "rare" are our only explanation for what has occurred. Yet, this does not seem to ameliorate the patient. The physician, in confronting the error, must first consider his or her own reaction and emotions before approaching the patient. Overwhelming guilt, frustration, and sadness need to be recognized. Discussion with a trusted colleague, social worker, or administrator can validate and 
alleviate some of the emotional anguish, which may inevitably create barriers to an honest and open approach with the patient. What should follow shortly thereafter is a conversation between doctor and patient - an admission of error.

First and foremost, patients seek acknowledgment of the error and the impact it will inevitably have on quality of life. Straight-forward explanations of how the error occurred, how it was discovered, and how the effects can be mitigated should be given. They will also seek a thoughtful description of the steps to be taken to prevent such errors from happening in the future. To hear the potential implications on quality of life, elucidated in simple terms, helps to prepare them for what is expected. An outline of the next treatment steps and the options going forward will help alleviate anxiety and fear of what may be to come. Active listening, reassurance of supportive services available, and direct communication with the teams who will help manage complications as they arise can help rebuild confidence in the team of doctors. Questions should be answered and concerns be addressed in order to meet the needs of the patient.

Perhaps, most importantly, patients deserve to hear an expression of apology in a way that demonstrates sensitivity to what they may be experiencing. Often, the doctor is reluctant to do this because of an ingrained belief that an apology is an admission of guilt, not just an acknowledgment of the patient's grief. Instead, the physician, wearing a yoke of perfection and untrained to confront the emotional consequences of the mistake, may try to shift blame to diffuse the severity of the error. This is counterproductive because patients find it difficult to regain trust in the physician-patient relationship in the absence of an apology. Furthermore, sitting with the inevitable tension and allowing the patient time to express his emotion can be the most meaningful demonstration of empathy. Compassion and expression of regret can help regain trust, which is the key to alleviating the patients' anger and bitterness directed against the physician for his suffering. We, as practitioners in an evolving field that emphasizes superior quality and elimination of human error through improved technology, would benefit from reflection and consideration of our own mental and emotional preparedness for an error. While no one ever looks forward to making use of a disaster kit, having one prepared certainly helps minimize further chaos and destruction.

\section{References}

1. Bogdanich, W. Radiation offers new cures, and ways to do harm. The New York Times. January 23, 2010. http://www.nytimes.com . Accessed 5 May 2014

2. Bogdanich W. Oncologist defends his work at a V.A. hospital. The New York Times. June 30, 2009. http://www.nytimes.com . Accessed 5 May 2014

3. Bogdanich W, Rebelo K. A pinpoint beam strays invisibly, harming instead of healing. The New York Times. December 28, 2010. http://www.nytimes.com . Accessed 5 May 2014 